Bundesgesundheitsbl 2009 $52: 375-376$

DOI 10.1007/s00103-009-0845-1

(C) Springer Medizin Verlag 2009

T. Sudhop ${ }^{1} \cdot$ H. Enzmann ${ }^{1} \cdot$ R. Klar ${ }^{2}$

${ }^{1}$ Bundesinstitut für Arzneimittel und Medizinprodukte, Bonn

${ }^{2}$ Institut für Medizinische Biometrie und Medizinische Informatik, Universitätsklinikum Freiburg

\title{
Klinische Forschung
}

\section{Auswirkungen der europäischen Gesetzgebung und aktuelle Entwicklungen}

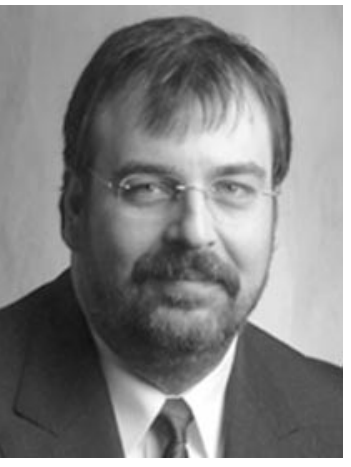

Thomas Sudhop

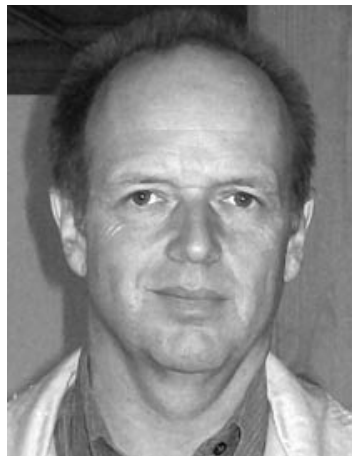

Harald Enzmann

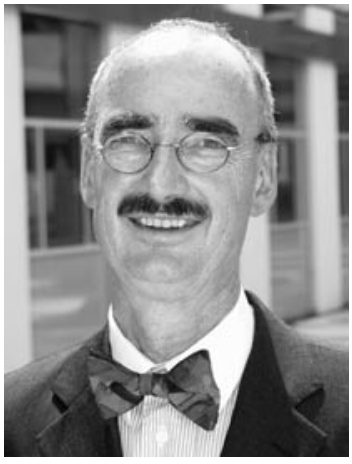

Rüdiger Klar in Deutschland wesentlich verschärft, sodass sich die Arbeiten im vorliegenden Heft zu einem Teil - aber nicht ausschließlich mit der klinischen Erforschung von Arzneimitteln am Menschen beschäftigen.

Janet Schriever berichtet zusammen mit ihren Koautoren über die Erfahrungen der beiden Bundesoberbehörden über das seit August 2004 geltende Genehmigungsverfahren für klinische Prüfungen von Arzneimitteln. Anhand verschiedener Statistiken können die Autoren zeigen, dass die initial gehegten Befürchtungen, die klinische Arzneimittelforschung käme durch die neue, aufwendigere auf der europäischen Clinical Trials Direktive (2001/20/EG) basierenden Gesetzgebung weitgehend zum Erliegen, nicht zutreffen. Aufgrund der nun erstmals generell vergleichbaren Bedingungen für die klinische Prüfung von Arzneimitteln in der EU zeigen sie, dass Deutschland schon kurz nach Einführung der neuen Regularien einen Spitzenplatz unter den Mitgliedsstaaten einnehmen konnte und seit mehr als zwei Jahren führend in der Anzahl der klinischen Prüfungen in Europa ist.

Durch die Umsetzung der Clinical Trials Direktive in nationales Recht änderten sich in Teilen die Aufgaben und Arbeitsweisen der Ethik-Kommissionen in Deutschland. Elmar Doppelfeld stell die Entwicklung der Aufgaben der medizinischen Ethik-Kommissionen nicht nur bei der beratenden Bewertung von klinischen Prüfungen, sondern auch bei Bewertung biomedizinischer Forschungsvorhaben am Menschen dar und berichtet über den Arbeitskreis der Medizinischen Ethik-Kommissionen, in dem sich alle 53 nach Landesrecht gebildeten EthikKommissionen zusammengeschlossen haben, um auffreiwilliger Basis die Arbeit der Kommissionen zu harmonisieren.

Jürgen Windeler und Stefan Lange beschäftigen sich in ihrem Aufsatz mit der Methodik klinischer Studien und der Interpretation von Studienergebnissen. Obwohl das Prinzip der klinischen Studie schon lange etabliert ist, ist die Verbesserung der verwendeten Methodik selbst heutzutage noch nicht abgeschlossen. Die Kenntnis besonders auch der modernisierten statistischen Studiendesigns erleichtert dem Arzt die Interpretation der Studienergebnisse und kann helfen, dass diese besser kommuniziert und besser verstanden werden.

Entscheidend für die Wahl eines Prüfzentrums ist neben der Qualität der Ein- richtung und der Qualifikation des Prüfers und des Prüfteams heute zunehmend die Fähigkeit eines Zentrums, geeignete Studienteilnehmer in vergleichbarer kurzer Zeit in eine wissenschaftliche Studie einschließen zu können. „Parallel recruitment" ist ein in der pharmazeutischen Industrie seit einigen Jahren gebräuchliches Verfahren, um möglichst rasch die gewünschte Anzahl von Studienteilnehmern durch eine Vielzahl konkurrierender Zentren zu erhalten; Zentren oder gar Länder, die nur geringe Rekrutierungsraten vorweisen, können so nur wenige - unter Umständen in den kurzen Rekrutierungsintervallen auch gar keinePatienten in klinische Prüfungen mit innovativen Arzneimitteln einschließen. Barbara Grün und Walter Haefeli beschäftigen sich in ihrem Aufsatz mit den verschiedenen Aspekten der Rekrutierung von Studienteilnehmern und liefern wertvolle Hinweise für erfolgreiche Rekrutierungsmaßnahmen in klinischen Studien.

Spätestens seit Inkrafttreten der Verordnung (EG) 1901/2006 des Europäischen Rates und Parlaments ist auch die pädiatrische Population mit ihrer extremen $\mathrm{He}$ terogenität in das tiefgehende Interesse der 
forschenden pharmazeutischen Industrie gerückt. Dieses auch als Kinderarzneimittelverordnung bezeichnete europäische Regelwerk macht erstmals die Forschung an Kindern und Jugendlichen verpflichtend im Rahmen der Entwicklung neuer Arzneimittel. Mit einer Mischung aus Verpflichtungen und Anreizen soll verhindert werden, dass neue Arzneimittel wie in der Vergangenheit nahezu ausschließlich für Erwachsene entwickelt werden. So müssen nun bereits während des klinischen Entwicklungsprogramms für Erwachsene Planungen für ein pädiatrisches Entwicklungskonzept (pädiatrisches Prüfkonzept) vorgelegt werden. Birka Lehmann und Dirk Mentzer stellen die wesentlichen Aspekte dieser neuen Regularien und Implikationen für die klinische Forschung an Minderjährigen vor, wobei wissenschaftliche, ethische und therapeutische Aspekte zu beachten sind. Schließlich wird auch die Rolle des Pädiatrieausschusses, einem neuen Gremium bei der Europäischen Arzneimittelagentur, das im Wesentlichen mit der Beurteilung pädiatrischer Prüfkonzepte betraut ist, erläutert.

Die Veränderungen des regulatorischen Kontextes von klinischen Arzneimittelstudien innerhalb der letzten fünf Jahre werden teilweise auch kritisiert. Nicola Gökbuget und Dieter Hölzer stellen in ihrem Aufsatz die Bedeutung von Multicenterstudiengruppen für die klinische Forschung in der Hämatologie und Onkologie in der Vergangenheit dar und beleuchten kritisch die aktuelle Situation der Therapieoptimierungsstudien als wichtigen Bestandteil der Versorgungsforschung.

Neben der therapeutischen Forschung sind aber auch Studien zur Bewertung diagnostischer Tests von zunehmendem Interesse. Ulrich Abel und Katrin Jensen beleuchten in ihrem Aufsatz die Methodik von Diagnosestudien und Diagnosetests und stellen anhand von anschaulichen Beispielen verschiedene Validitätskriterien vor und erläutern analog zur Arzneimittelprüfung die vier Phasen einer Diagnosestudie. Neben Diagnosestudien werden auch andere klinische Studien außerhalb des Gültigkeitsbereichs des Arzneimittelgesetzes durchgeführt. Dies trifft zum Beispiel für einen Großteil der klinischen Studien in der Chirurgie zu. Christoph Seiler und Kollegen beleuchten in ihrem Aufsatz moderne Methoden in der chirurgischen klinischen
Forschung und zeigen Parallelen wie Unterschiede zur Methodik der klinischen Arzneimittelprüfung.

In den letzten Jahren haben sich die klinischen Entwicklungszeiten für neue Arzneimittel insbesondere bei lebensbedrohlichen Indikationen bereits leicht verkürzt. Wo sinnvoll und nötig kann darüber hinaus auch schon während der Entwicklung - also noch vor der Zulassungsentscheidung Patienten Zugang zu neuen Arzneimitteln ermöglicht werden. Mit einem bestimmten Aspekt der Abgabe noch nicht zugelassener Arzneimittel, dem sogenannten Compassionate Use, setzt sich der Aufsatz von Thomas Sudhop auseinander. Compassionate Use, wörtlich übersetzt „der barmherzige Einsatz", bezeichnet regulatorisch die Abgabe eines in der klinischen Entwicklung befindlichen Arzneimittels vor der eigentlichen Zulassung an bestimmte Patienten. Der Artikel beschäftigt sich mit den rechtlichen und medizinischen Voraussetzungen eines solchen Arzneimitteleinsatzes, soweit diese bisher regulatorisch absehbar sind.

Die Anforderungen an die Durchführungsqualität klinischer Prüfungen von Arzneimitteln sind mit der Clinical Trials Direktive 2001/20/EG und der Umsetzung in nationales Recht durch die 12. AMGNovelle weiter gestiegen. Um auch Universitäten in die Lage zu versetzen, in diesem komplexen regulatorischen und organisatorischen Umfeld weiterhin klinische Prüfungen von Arzneimitteln durchführen zu können, wurden an ausgewählten medizinischen Fakultäten Koordinierungszentren für klinische Studien (KKS) eingerichtet. Steffen Luntz und seine Kolleginnen und Kollegen der Koordinierungszentren der Universitäten Heidelberg und Mainz beschreiben in ihrem Aufsatz die Entwicklung und Aufgaben solcher Zentren und stellen anhand des breiten Portfolios an Dienstleistungen dar, wie ein KKS sowohl rein universitäre wie auch Drittmittel-finanzierte Forschung unterstützen kann. Das seit vielen Jahren bestehende nationale Netzwerk der Koordinierungszentren für klinische Studien (KKS-Netzwerk) wird von Insa Bruns, Stephanie Wolff und Herbert Maier-Lenz vorgestellt und in seiner Funktion für den Forschungsstandort Deutschland gewürdigt.

Der letzte größere Schwerpunkt des Heftes beschäftigt sich mit dem neu einge- richteten nationalen Register für klinische Studien. Gert Antes und Koautoren führen mit ihrem Aufsatz in das Thema ein und beleuchten die Hintergründe der Entwicklung des nationalen Registers für klinische Studien. Vor allem gilt es, auch nicht erwünschte oder vermeintlich uninteressante Studienergebnisse bekannt zu machen, um für eine evidenzbasierte Medizin den Publicationbias zu verringern. Gabriele Dreier und Koautoren stellen dazu das frei zugängliche Deutsche Register Klinischer Studien vor, das den Anforderungen der WHO und des International Committee of Medical Journal Editors genügt.

Wir hoffen, mit dieser Auswahl an Themen breite aktuelle Aspekte der klinischen Forschung in Deutschland angesprochen zu haben und wünschen Ihnen bei der Lektüre viel Vergnügen.

Ihre

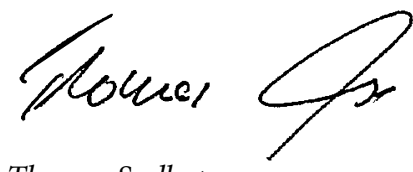

Thomas Sudhop

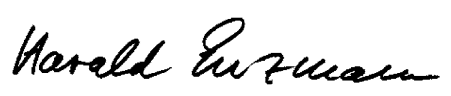

Harald Enzmann

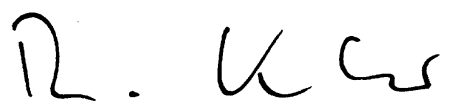

Rüdiger Klar

\section{Korrespondenzadresse \\ PD Dr. T. Sudhop}

Bundesinstitut für Arzneimittel

und Medizinprodukte

Kurt-Georg-Kiesinger-Allee 3, 53175 Bonn

sudhop@bfarm.de 